measured on the SF-36. In general, depression was not associated with a diagnosis of either asthma or COPD.

Conclusions: The prevalence of depression was higher than in previous studies. Importantly, depression was more common in people with significant health risk factors but not with chronic lung diseases. Regular exercise was associated with a reduction in rates of depression. The possible reasons for this will be examined.

\section{Stress Sampler I: psychosocial adjustment and psychiatric and physical comorbidity in diabetes mellitus}

\section{J Reddy ${ }^{1,2}$, L Wedgwood ${ }^{3}$, J Siegel ${ }^{3}$, T Showyin ${ }^{3}$, A Finch ${ }^{3}$, K Baikie ${ }^{3}$, G Parker ${ }^{3}$, P Schofield ${ }^{4}$, P Mitchell2 ${ }^{2}$ K Wilhelm ${ }^{1,3}$}

'St Vincent's Hospital: 2 School of Psychiatry, University of New South Wales; ${ }^{3}$ The Black Dog Institute, Sydney, Australia; and 4Prince of Wales

Medical Research Institute, Sydney, Australia

Background: Diabetes mellitus is a chronic illness that causes significant psychological and physical morbidity. The psychological needs of patients with diabetes mellitus are often undetected and neglected. This paper describes preliminary data from the Stress Sampler Study designed to trial brief psychological interventions to improve psychological and physical health among patients with type I and type II diabetes mellitus. We describe psychosocial adjustment specific to diabetes mellitus and its association with psychiatric and physical comorbidity.

Methods: The Stress Sampler Study, commenced in July 2006, has been recruiting patients presenting to a hospital-based specialist diabetes clinic. Patients are administered the Patient Health Questionnaire, the Problem Areas in Diabetes Survey, SF-12 (physical and psychological self-report of functioning) and a clinical interview by a qualified psychiatrist as part of baseline assessment.

Results: We present preliminary data from the first 100 patients examining the usefulness of a specific diabetes-related measure of psychosocial adjustment and its association with psychiatric diagnosis and physical comorbidity.

Conclusions: These findings will contribute to the identification of specific psychological needs and problem areas among patients with diabetes. Knowledge of specific needs will greatly assist clinicians and researchers in the process of development of specific psychological interventions for patients with diabetes mellitus.

\section{Exploring depressive symptoms in mid-adolescence: the integrated effects of life events and personality}

\author{
N Reece, S Thomson, K Jarman, A Onley, N Turner, \\ R Brown
}

University of Newcastle, Newcastle, Australia

Background: There is ample evidence that adolescent depressive symptoms are a precursor of depression in later life (Canals et al. 2002; Pine et al. 1999). The period of mid-adolescence is especially significant because it is regarded as a time of major developmental changes and challenges (Kardum \& Krapic 2002). Although the majority of teens exhibit considerable resilience, the prevalence of depressive symptoms greatly increases during this transitional period, and there are several factors shown to create vulnerability in certain individuals.

Methods: This study explored an integrated model to investigate the possible moderating relationship between personality characteristics and stressful life events associated with a vulnerability to depression within mid-adolescence (Kardum \& Krapic 2001). Using a retrospective design, 324 year 9 adolescents from Maitland-Newcastle high schools completed the Eysenck Personality Questionnaire-Junior, Adolescent Life Change Event Questionnaire and Centre for Epidemiological Studies-Depression Scale for Children.

Results: As expected, personality and life events were correlated with depressive symptomatology and female adolescents scored higher on most measures. Significant interactions indicate that although life events can predict depressive scores, neuroticism has a greater influence independently and by moderating the effects of stressful life events on depressive symptoms. Additionally, specific life events, such as appearance problems, may impact greater on adolescents' emotional adjustment.

Conclusions: Results suggest modest support for an integrated model in explaining depressive symptoms in mid-adolescence and indicate possible risk factors rather than causal relationships. Results would be enhanced by a prospective design.

\section{Insensitivity to temporal context in schizophrenia}

\author{
A Richards, J Todd, P Michie \\ The University of Newcastle, Australia
}

Background: Elicitation of the mismatch negativity (MMN) occurs when the auditory system detects 